\title{
Morphological changes in textile fibres exposed to environmental stresses: Atomic force microscopic examination
}

\author{
Elisabetta Canetta ${ }^{\ddagger}$ Kimberley Montiel, Ashok K. Adya* \\ Condensed Matter Group and BIONTHE $\left.\right|^{\dagger}$ Centre, \\ Division of Biotechnology and Forensic Sciences, School of Contemporary Sciences, \\ University of Abertay Dundee, Bell Street, Dundee DD1 1HG, Scotland, U. K.
}

\begin{abstract}
The ability of the Atomic Force Microscope (AFM) to investigate the nanoscopic morphological changes in the surfaces of fabrics was examined for the first time. This study focussed on two natural (cotton and wool), and a regenerated cellulose (viscose) textile fibres exposed to various environmental stresses for different lengths of time. Analyses of the AFM images allowed us to measure quantitatively the surface texture parameters of the environmentally stressed fabrics as a function of the exposure time. It was also possible to visualise at the nanoscale the finest details of the surfaces of three weathered fabrics and clearly distinguish between the detrimental effects of the imposed environmental conditions. This study confirmed that the AFM could become a very powerful tool in forensic examination of textile fibres to provide significant fibre evidence due to its capability of distinguishing between different environmental exposures or forced damages to fibres.
\end{abstract}

Keywords: Atomic force microscopy; Textile fibres; Cotton; Wool; Viscose; Surface Texture

\footnotetext{
* Corresponding author: a.k.adya@abertay.ac.uk, Tel.: +44-(0)-1382-308653, Fax: +44-(0)-1382-308663

$\$$ School of Physics and Astronomy, University of St Andrews, North Haugh, St Andrews, Fife, KY16 9SS

${ }^{\dagger}$ Bio- and Nano-Technologies for Health and Environment
} 


\section{Introduction}

Textile fibres play a fundamental role in our daily life. They are ubiquitous and can be found in clothes, household textiles, carpets, floor coverings, curtains, and upholstery.

Since textile fibres can be involved in many crimes, a correct and accurate characterisation of their morphological, physical, and chemical features is essential in solving murder, sexual offence, arson, and burglary cases [1]. Textile fibres are classified into two basic groups: natural fibres (i.e., cotton, wool, and asbestos), and man-made fibres (e.g., viscose and polyester) [1].

Forensic fibre analysts are quite often asked to examine fibres found outside (e.g., in different types of soil and water), or on a variety of outdoor surfaces (e.g., park benches, roof tiles, paving stones). A survey of the significance of fibres on outdoor surfaces has shown that cotton and viscose are the textile fibres most commonly found outside, with only a small fraction of wool probably due to the fact that wool fibres degrade more easily [2]. When a fibre lies in an outside environment for short or long periods of time, it is subjected to a variety of environmental stresses, such as sunlight, rain, soil burial, water immersion. Investigating the morphological changes of environmentally stressed fibre surfaces and the changes in their physical (e.g., adhesion to a surface) and mechanical (e.g., elasticity) properties can be essential in understanding when and where a crime was committed.

Generally, a forensic examination concerns the comparison of fibres found at a crime scene with a variety of different known fibres in order to understand where the suspect fibres came from. During this comparison/elimination process the major goal is to achieve a very high degree of discrimination between very similar samples by analysing the morphological, physical, and chemical features of the suspect fibres [1]. With this aim in view different techniques are used, such as microscopy, 
photodiodearray spectrophotometery (PDA); microspectrophotometry, thin layer chromatography (TLC) and surface enhanced resonance Raman scattering (SERRS); infrared, FTIR (Fourier Transform Infrared), Raman microspectroscopy; and pyrolisis gas chromatography $[1,3]$. These techniques are used e.g., for morphological investigation, colour/dye characterisation, synthetic fibres and fibre additives identification. Among the microscopic techniques routinely used in a forensic laboratory, the most common are optical, fluorescence, and comparison microscopy. Scanning electron microscopy (SEM) has also been employed as an imaging tool. However, SEM is a destructive technique because the sample surface has to be treated to make it conductive prior to visualisation and the specimens are desiccated by the vacuum conditions. With the advent of environmental SEM (ESEM) these problems have been overcome, though much research is still needed to study a host of others phenomena that may arise, such as interactions of gas, radiation and hydrated samples to generate gaseous by-products and mobile free radicals that can interact with the specimen, with yet unknown consequences. These changes may or may not become visible, but user should be aware of the possible existence of such effects during the evaluation of results. Nevertheless, one drawback common to both SEM and ESEM is that these techniques do not provide any information on heights and roughness of fibre surface. These data can sometimes provide additional valuable information in the discrimination (comparison/elimination) process.

Recently, atomic force microscopy (AFM) [4] has been employed to image at the nano-scale the surfaces of different samples of interest in forensic examinations, such as hair [5, 6], line crossings [7], latent fingerprints on metallic surfaces [8], bloodstains [9], cold plasma treated PET (polyethyleneterephthalate) fabrics [10], and bio-scoured cotton fabrics [11]. Although AFM cannot image large (e.g. few mm) 
samples compared to optical microscopes or SEMs, its capability to visualise nanoscopic morphological details by generating 2D and 3D images of the specimen, such as textile fibre surfaces, in their natural state and to quantify surface texture parameters, such as heights and roughnesses, can provide complementary and additional valuable information to characterise them with a high level of confidence. It is possible to discriminate between samples that at the macro- or micro-level may look similar. Its non-destructive nature, very high resolution, and capability to detect extremely subtle differences in the morphology of the samples makes AFM a very attractive and useful technique in forensic science applications. However, only little work has been done so far to investigate the morphology of fibres by using this technique. Other strengths of AFM lie in its versatility to study the physical, mechanical, and chemical properties of the sample, such as its adhesion to a substrate and its elasticity. In particular, investigating the elastic features of textile fibres could be beneficial to discriminate between different types of fibres. AFM can, therefore, be considered as a fast emerging complementary technique to optical and electron microscopic methods and chemical assays routinely used in any forensic laboratory. It is worth stressing, however, that much more work still need to be done before it can be claimed that this technique is capable of discriminating between false negatives or false positives.

Nevertheless, we conclusively demonstrate in this study that AFM can be successfully used to investigate the morphological changes on the surfaces of different environmentally stressed or weathered textile fibres. 


\section{Materials and methods}

\section{Textile fibre samples and environments}

Three different textile fabrics, one of each type, were studied: two natural (cotton and wool), and one man-made (viscose). The samples were bought from Shingar Fabric Shop, Dundee (UK). Cotton was mercerised while wool was carded. The size of each fabric used was about $6 \mathrm{~cm}^{2}$.

The environments chosen to weather the textile fabrics were: $(a)$ loam soil (obtained from the Scottish Crop Research Institute, SCRI, Dundee), (b) riverside soil (obtained from the riverside of the Tay, Dundee), (c) pond water (obtained from an artificial lake used for crop irrigation at the SCRI, Dundee), and (d) sea water (obtained from the North Sea, Broughty Ferry, Dundee).

\section{pH measurements of each environment}

The $\mathrm{pH}$ of each environment (the soils and waters) without any fabric inside (week 0), and the soils and waters with the cotton, wool, and viscose samples kept inside for 6 weeks were measured every two weeks for up to 6 weeks.

\section{Environmental stresses}

The three different types of fabrics were exposed to different environmental conditions by burying them in the loam and riverside soils, and immersing them in the pond and sea waters. The fabric samples were taken out for AFM imaging every 2 weeks for up to 6 weeks. The fabrics not exposed to any environmental stress (week 0) were used as a control.

For the soil burial, $3 \mathrm{~cm}$ thick layer of loam or riverside soils were transferred into several garden trays. The fabric samples were then spaced evenly into each garden tray and covered with another $3 \mathrm{~cm}$ thick layer of the same type of soil. The trays were kept at room temperature with equal access to light and oxygen. The soils were 
sprayed with approximately $50 \mathrm{ml}$ of distilled water 3 times a week to avoid the soil to dry out. After taking out the samples, they were cleaned by removing the soil with a soft, dry brush.

For the water immersion, each textile fabric sample was immersed in covered tubs containing approximately $200 \mathrm{ml}$ of pond or sea water. To avoid a quick evaporation of the water, the tubs were stored in a dark cupboard at room temperature. In order to ensure that the textiles were equally exposed to the water, the tubs were shaken for 10 minutes twice a week by using an orbital shaker $(216 \mathrm{rpm}=11 \mathrm{~g})$. When the samples were taken out, they were left to dry overnight at room temperature.

\section{Atomic force microscopy (AFM)}

The surface topography of the three different environmentally stressed fabrics along with their controls was imaged by using an atomic force microscope (AFM) [4].

The AFM used was a NanoWizard ${ }^{\circledR}$ I BioAFM (JPK Instruments AG, Berlin, Germany). It was operated in contact mode. Au-coated $\mathrm{Si}_{3} \mathrm{~N}_{4}$ cantilevers, with a nominal spring constant of 0.03 N/m (Veeco Probes, Santa Barbara, CA), were used. All the AFM experiments were performed in air at room temperature, and the height images $(10 \mu \mathrm{m} \times 10 \mu \mathrm{m}$ in size and $512 \times 512$ pixels $)$ were collected using a scan speed of $0.5 \mathrm{~Hz}$. Since the size of the AFM image was larger than $1 \mu \mathrm{m}^{2}$, its lateral resolution was determined not by the radius of the tip but by the step size. A lateral resolution of $20 \mathrm{~nm}$ for all the captured images was estimated.

The surface of each fabric was scanned in the $x, y$, and $z$ directions by a sharp tip micro-fabricated on the cantilever, and moved by a piezoelectric translator. A laser beam reflected off the cantilever towards a 4-segment photodiode sensed the deflection undergone by the cantilever when the tip scanned the sample surface. The interaction force between the tip and the sample could be directly obtained from the 
cantilever deflection using the Hooke's law $(F=k \cdot x$, where $k$ is the spring constant of the cantilever and $x$ its deflection). The oscillation amplitude was used as input for a feedback circuitry that allowed us to maintain the tip at a constant $z$ distance from the sample surface. The curve $z=f(x, y)$ provided the surface topography (i.e., the height image) of the fabric specimens.

To prepare the AFM samples, a single fibre from each of the textile samples unexposed (control) and exposed to different environmental conditions was mounted onto a glass slide using adhesive tape, ensuring that the fibre was straight and strongly adhered to the slide. To obtain repeatable results measurements were repeated for three different individual fibres for each fabric. The AFM images of three different areas on each of the three individual unexposed (control) and environmentally exposed fabrics were analysed, and the heights and roughnesses measured to establish and explain the observed trends.

\section{Surface texture analysis}

The surface texture parameters $[10,11]$ of the textile fibres evaluated in this study were the following:

(a) average maximum peak heights $\left(H_{p m}\right)$;

(b) average maximum heights $\left(H_{z}\right)$;

(c) average maximum valley depths $\left(H_{v m}\right)$;

(d) peak-to-valley distances $\left(R_{z}\right)$, and

(e) root mean square roughnesses $\left(R_{r m s}\right)$.

The surface texture parameters (a) - (d) were evaluated by carrying out section analyses, while the parameter (e) was obtained by performing roughness analysis, on the AFM height images by using the JPK image-processing software (JPK Instruments AG, Berlin, Germany). 
The average maximum peak heights, $H_{p m}$, and the average maximum valley depths, $H_{v m}$, of the investigated fibres were obtained by averaging, respectively, the heights and depths of 40 higher peaks and lower valleys found on the complete AFM height image. For a more reliable identification of the peaks and valleys on a fibre surface, the 3D AFM image was used as a reference while performing the section analysis on the corresponding AFM height image (see Fig. 1 as an example). The AFM height images were filtered with a high pass filter to sharpen the images prior to section analysis in order to obtain reliable results.

The average maximum heights, $H_{z}$, were calculated by using [11],

$$
H_{z}=H_{p m}-H_{v m}
$$

where $H_{p m}$ and $H_{v m}$ are the average maximum peak height and the average maximum valley depth, respectively.

The peak-to-valley distances (or nominal roughnesses, $R_{z}$ ) were measured by performing section analysis on 100 different positions of each textile surface. The values of the peak-to-valley depths were then treated statistically and the average of these values calculated.

The roughness of the surface of each fibre was also analysed by measuring the root mean square roughness, $R_{r m s}$, on the AFM height image, defined as the standard deviation from the mean data plane of the $H$ (height) values of the AFM images within a selected region on the fibre surface,

$$
R_{r m s}=\sqrt{\frac{\sum_{i=1}^{N}\left(H_{i}-\bar{H}\right)^{2}}{N}} .
$$

In Eq. (2), $H_{i}$ is the current height value, $\bar{H}$, the height of the mean data plane, and $N$, the number of points within the selected region of a given area. The roughness analysis was carried out on the low-pass-filtered AFM height images. The $R_{r m s}$ was 
evaluated over 50 different areas of each scanned fibre surface. The areas chosen were $2 \mu \mathrm{m}^{2}$. The average of these roughness values was then calculated.

The results for the surface texture parameters, $H_{p m}, H_{v m}$ and $H_{z}$, are reported in Table 2, and those for $R_{r m s}$ and $R_{z}$ in Table 3, as mean \pm standard deviation values. The value of standard deviation was the criteria chosen to evaluate the repeatability of the results, and in most cases it was found to be $\sim \pm 7 \%$.

\section{Results and Discussion}

Table 1 lists the pH values at week 0 (control) and week 6 of two different types of soils and waters that were used to expose the three fabrics to different environments. The $\mathrm{pH}$ stayed nearly constant within $< \pm 0.5$ for each environmental condition and fibre during the entire duration of 6 weeks. This simply reflects that any change in the morphology that occurred due to the imposed environmental stress was not due to the $\mathrm{pH}$ change.

Figs. 2-4 present the AFM height images of unexposed ( 0 weeks) and environmentally exposed cotton, viscose, and wool fabrics after 2 and 6 weeks. The surfaces of all the three unexposed fibres appeared flatter and smoother than those of the exposed ones (Figs. 2-4). In particular, the different environmental conditions created pits and cavities in the fibres whose depths and sizes increased as a function of the exposure time.

Changes in the morphology of the weathered fibres were quantified (Tables 2 and 3) by section and roughness analyses (see Section 1: surface texture analysis) of the AFM height images. 


\subsection{Cotton}

Cotton is a vegetable fibre that is harvested from the seeds of several species of the genus Gossypium. It is a staple fibre whose surface is flat and twisted [1] as the AFM images of the unexposed cotton fibre clearly showed (Fig. 2). The twisted nature of the cotton surface is the main reason why cotton usually absorbs a lot of water and moisture.

The section analyses (Table 2) carried out on the AFM images of the weathered cotton fibres showed that the heights of the fabric exposed to different environments compared to the height, $\mathrm{H}_{\mathrm{z}}$, of the control ( 0 weeks), increased with the exposure time. This indicated that the environmentally stressed cotton fabric swelled as a function of the exposure time, probably due to the high absorbing power of cotton. In particular, when immersed in sea water, the height of the fabric increased from $36 \%$ ( 2 weeks) to $\sim 188 \%$ (6 weeks) (see Table 2) as compared to that of the control sample. In comparison, the exposure of the cotton fabric to riverside soil led to substantially higher increase in the heights of the fibres (2 weeks: 95\%, 6 weeks: $\sim 376 \%$, see Table 2). A further enormous increase in the height of the environmentally stressed cotton fabric occurred when it was immersed in pond water (2 weeks: $221 \%$, 6 weeks: ( 498\%, see Table 2$)$. The maximum swelling (highest damage to the fabric measured in terms of the height of cotton) was found when the fabric was stressed with the loam soil ( 2 weeks: $260 \%, 6$ weeks: $\sim 569 \%$, see Table 2 ). The AFM images (Fig. 2) clearly showed the detrimental effects, seen as deep and large cavities, caused by sea water and riverside soil on the surface of the exposed cotton after 6 weeks. Pond water did not affect dramatically the morphology of the cotton surface, as also shown by the AFM images (Fig. 2), though after 6 weeks of immersion of the cotton fabric in pond water a noticeable change in the surface 
morphology was visible. The least detrimental effects on the cotton surface morphology were those caused by loam soil as clearly shown by the AFM images after 6 weeks, and quantitatively confirmed by the roughness analysis (Table 3). The surface roughness of the cotton fibre exposed to loam soil was the lowest, while that of the fibre immersed in sea water was the highest.

The above results show that the increase in average maximum height, $\mathrm{H}_{\mathrm{z}}$, as compared to the control sample, follows the following order: loam soil $>$ pond water $>$ riverside soil $>$ sea water. However, the increase in fibre surface roughness decreases in the order: sea water $>$ riverside soil $>$ pond water $>$ loam soil, and this order is exactly the reverse of the order observed above for the increase in the average maximum heights.

\subsection{Viscose}

Although viscose is a man-made regenerated cellulosic fibre, its morphology is very similar to that of cotton. This clearly was confirmed by comparing the AFM images of the unexposed cotton (Fig. 2) and viscose (Fig. 3) fibres. Since the nature of the viscose polymer chain is amorphous and the number of polar hydroxyl groups is very large, viscose is even more absorbent than cotton [1]. This feature was confirmed by the section analyses performed on the AFM images of the environmentally stressed viscose (Table 2) that showed that the lowest increase in height value was obtained when viscose was immersed in sea water. Nevertheless, the behaviour of viscose differed from that of cotton when exposed to the other environmental stresses.

The results obtained show that viscose swelled more than cotton when exposed to different environmental stresses, its height being $\sim 83 \pm 55 \%$ (averaged over four different stresses exposed to different times over a six week period) higher than that 
of cotton. The surface roughness of viscose fibre, as expected, is also found to be higher than that of cotton (see Table 3).

Similar to cotton, sea water affected more than the other environmental stresses the morphology of viscose surface (Fig. 3). On the contrary, pond water was found to be the least environmental condition to affect the surface of viscose, as also clearly shown by the AFM images (Fig. 3). Because it caused the maximum swelling of the fibre, the roughness of its surface was lower than that of the viscose fibres exposed to any other environmental stress.

The results for the section and roughness analyses (see Tables 2 and 3) show that the increase in average maximum heights, $\mathrm{H}_{\mathrm{z}}$, as compared to the control samples decreases in the order: pond water $>$ riverside soil $>$ loam soil $>$ sea water, and this order is different from that for the cotton fabric. However, the increase in roughness values follows the order: sea water $>$ loam soil $>$ riverside soil $>$ pond water, and this order is exactly the reverse of that observed for the increase in the average maximum heights. Interestingly enough, similar reversal of trend was observed (see above) for the cotton fabric. This simply adds credence to the analyses procedures employed in the present work, and we shall see whether the same observation holds for the wool fabric.

\section{$2.3 \mathrm{Wool}$}

Wool is an animal fibre that is produced in the fibre follicle in the skin of the sheep. It is a staple fibre with a rough scaly surface [1] as also confirmed by the AFM images of the unexposed wool fibre in which it was possible to distinguish the three scales (Fig. 4). Unlike cotton and viscose, wool does not have a fixed and standard morphology. The main morphological characteristics commonly used to distinguish 
between different kinds of wool fibres are the diameter, the scale thickness, scale prominence, and scale count of the fibres.

Wool's behaviour was found to be different from that observed (see Sections 2.1 and 2.2 above) for both cotton and viscose that differ from each other in showing different trends for different environmental stresses. The heights of wool fabrics exposed to different environmental conditions decreased (See Table 2) showing that while cotton and viscose swelled on environmental exposures, the wool shrank. This was also clear from the AFM images in which the disruption and/or flatness of the three scales could be observed (Fig. 4). In particular, when wool was exposed to the two types of soil, the surface of the fibre became extremely flat and rough, as shown by section and roughness analyses, and the pattern observed in the unexposed wool fibre disappeared completely (Fig. 4). Immersion of wool in the two different kinds of water affected the surface of the exposed fibre slightly less than the effect caused by exposure of the fabric to two different types of soil. Sea water was more detrimental than pond water to the morphology of the environmentally stressed wool, the three scales observed in the unexposed wool fibre were still visible after 2 (Fig. 4) and 4 (image not shown) weeks of immersion in pond water. This was also confirmed by section and roughness analyses that showed shrinkage of the wool surface, and thus an increase in the roughness of the surface when wool was exposed to sea water.

The results show (see Table 2) that the increase in average maximum heights, $\mathrm{H}_{\mathrm{z}}$, as compared to the control samples, decreased with different environmental stresses on the wool fabric in the order: pond water $>$ sea water $>$ riverside soil $>$ loam soil. Interestingly, the results show an exact reversal of trend in going from average maximum heights, $\mathrm{H}_{\mathrm{z}}$, to surface roughness (see Table 3), and this trend is similar to the ones observed above for both cotton and viscose. Although different surface 
texture parameters are used in different contexts to describe surface roughness of materials, such as engineering components, it is difficult to comprehend such a consistent reversal of order of the three different weathered fibres exposed to varying environmental conditions as observed in this study. From the present study alone, one can only conjecture that this may possibly be due to the fact that when the fibres are weathered their surfaces wrinkle and crack causing a decrease in the height, and therefore resulting in an increase in their surface roughnesses.

The detrimental effects (measured in terms of increase in height, $\mathrm{H}_{\mathrm{z}}$ ) of different environmental stresses on the morphology of the three fabrics in the decreasing order: loam soil $>$ pond water $>$ riverside soil $>$ sea water (for cotton), pond water $>$ riverside soil $>$ loam soil $>$ sea water $($ for viscose), pond water $>$ sea water $>$ riverside soil > loam soil (for wool), were found to be different for different fibres. The above results show that for any particular type of environmental condition, the longer the exposure time the more is the damage done to any type of fabric. Also, the degree and the trend of detrimental effect of different environmental exposure are found to vary from fabric to fabric. Nevertheless, the results conclusively show that by using the AFM technique, it is possible to quantify the damage caused by an environmental stress to any fibre.

From the analysis carried out in this study, we are of the view that $\sim 40$ analyses of the heights (higher peaks and lower valleys) and 100 positions analysed for the surface roughness on each of the 3 different areas on 3 separate fibres of the same fabric recovered typically in a forensic casework should provide representative data of the analysed samples, and the value of standard deviation of $\sim \pm 7 \%$ should be considered sufficient for evaluating the repeatability of the results. 


\section{Conclusions}

AFM has been used previously to investigate changes in the morphology of the fabric surfaces, which have undergone physical (e.g., plasma) [10] and chemical (e.g., bioscouring) [11] changes. In the present work, AFM's ability to examine quantitatively the nanoscopic morphological changes in the surfaces of environmentally stressed fibres has been explored.

The AFM allowed us to visualise at the nanometer resolution the finest details of the surfaces of three different fibres (cotton, viscose, and wool) subjected to several environmental conditions for different length of times. In addition, the heights and roughnesses of the unexposed and exposed fibre surfaces were measured by analysing the AFM images.

Bearing in mind that only one type of each fabric was studied in this work, recognisable nanoscopic differences in the morphology of the three weathered fabrics could be clearly observed and quantified. Cotton was most affected by the environmental stresses, with the effects of sea water (loam soil) being most (least) detrimental to the morphology of its surface. Similar to cotton, viscose was also highly affected by sea water, though slightly less than cotton. However, the least detrimental environment condition to affect the surface of viscose fibre was pond water. Wool seemed to be the least affected by all the environmental conditions in comparison to cotton and viscose. Similar to viscose, the effects of pond water on the morphology of wool fibre were the least dramatic, though these effects were even smaller than those observed for viscose. The morphology of wool fibre appeared to be affected most by the loam soil.

The above results further confirm the potential use and applicability of AFM in forensic examination of fibres because of its non-destructive nature (the fibres do not 
get damaged or destroyed by the AFM scanning), very high (nanometer scale) resolution, and the possibility of accurately interpreting the data. AFM can, thus, be usefully included amongst the other techniques commonly employed in forensic sciences and viewed as a complimentary technique to optical microscopy, SEM, and ESEM.

In this work, we used the AFM only as an imaging tool, but AFM has also the potential to be employed in force spectroscopy mode to study the physical (e.g., adhesion to a surface) and mechanical (e.g., elasticity) properties of fibres. This information on adhesive and elastic properties, along with the finest details (2D height and 3D AFM images) of the fibre surfaces, surface roughness and other surface texture parameters could be usefully employed to compare different types of fibres, and ascertain the damages caused by crimes and environmental exposures. Nevertheless, this field needs to be further explored, and the present studies form a first step in this direction.

\section{Acknowledgements}

We wish to acknowledge the SHEFC for the award of a SRIF II grant to purchase the JPK AFM, and the University of Abertay Dundee (UAD) for the support of this work. One of us, EC, is grateful to the UAD for the award of a postdoctoral research assistantship (PDRA). 


\section{Tables}

Table 1

$\mathrm{pH}$ values of the two types of soils and waters at week 0 (control) and week 6

\begin{tabular}{ccccc}
\hline \multirow{2}{*}{ Environment } & \multicolumn{5}{c}{$\mathrm{pH}$} \\
\cline { 2 - 5 } & Control & Cotton & Wool & Viscose \\
& $($ Week 0) & (Week 6) & (Week 6) & (Week 6) \\
\hline Loam soil & 7.5 & 6.7 & 6.9 & 7.0 \\
Riverside soil & 6.2 & 6.3 & 5.6 & 5.9 \\
Pond water & 6.9 & 7.6 & 7.3 & 7.8 \\
Sea water & 7.9 & & & 7.8 \\
\end{tabular}


Table 2

Average maximum peak heights $\left(\mathrm{H}_{\mathrm{pm}}\right)$, average maximum valley depths $\left(\mathrm{H}_{\mathrm{vm}}\right)$, and average maximum heights $\left(\mathrm{H}_{\mathrm{z}}\right)$. The values for $\mathrm{H}_{\mathrm{pm}}$, $\mathrm{H}_{\mathrm{vm}}$ (calculated over 40 higher peaks and lower valleys on the AFM image, respectively) and $\mathrm{H}_{\mathrm{z}}$ (Eq.(1)) are given as the mean \pm standard deviations.

\begin{tabular}{|c|c|c|c|c|c|c|c|c|c|c|}
\hline \multirow{2}{*}{ Time (weeks) } & \multirow{2}{*}{ Environment } & \multicolumn{3}{|c|}{ Cotton } & \multicolumn{3}{|c|}{ Wool } & \multicolumn{3}{|c|}{ Viscose } \\
\hline & & $\mathrm{H}_{\mathrm{pm}}(\mathrm{nm})$ & $\mathrm{H}_{\mathrm{vm}}(\mathrm{nm})$ & $\mathrm{H}_{\mathrm{z}}(\mathrm{nm})$ & $\mathrm{H}_{\mathrm{pm}}(\mathrm{nm})$ & $\mathrm{H}_{\mathrm{vm}}(\mathrm{nm})$ & $\mathrm{H}_{\mathrm{z}}(\mathrm{nm})$ & $\mathrm{H}_{\mathrm{pm}}(\mathrm{nm})$ & $\mathrm{H}_{\mathrm{vm}}(\mathrm{nm})$ & $\mathrm{H}_{\mathrm{z}}(\mathrm{nm})$ \\
\hline 0 & - & $0.88 \pm 0.01$ & $0.46 \pm 0.02$ & $0.42 \pm 0.03$ & $2.21 \pm 0.02$ & $0.72 \pm 0.04$ & $1.49 \pm 0.06$ & $1.04 \pm 0.01$ & $0.31 \pm 0.04$ & $0.73 \pm 0.05$ \\
\hline 2 & & $2.41 \pm 0.03$ & $0.91 \pm 0.06$ & $1.51 \pm 0.09$ & $1.61 \pm 0.04$ & $0.56 \pm 0.06$ & $1.04 \pm 0.11$ & $3.02 \pm 0.02$ & $0.72 \pm 0.06$ & $2.08 \pm 0.08$ \\
\hline 4 & Loam soil & $3.21 \pm 0.04$ & $1.04 \pm 0.04$ & $2.16 \pm 0.08$ & $1.22 \pm 0.04$ & $0.51 \pm 0.06$ & $0.71 \pm 0.11$ & $3.44 \pm 0.03$ & $0.82 \pm 0.04$ & $2.39 \pm 0.07$ \\
\hline 6 & & $4.01 \pm 0.08$ & $1.21 \pm 0.06$ & $2.81 \pm 0.14$ & $0.94 \pm 0.03$ & $0.37 \pm 0.03$ & $0.57 \pm 0.06$ & $4.45 \pm 0.06$ & $0.85 \pm 0.07$ & $3.15 \pm 0.13$ \\
\hline 2 & & $1.45 \pm 0.02$ & $0.63 \pm 0.04$ & $0.82 \pm 0.06$ & $1.81 \pm 0.04$ & $0.61 \pm 0.05$ & $1.21 \pm 0.09$ & $3.21 \pm 0.05$ & $0.83 \pm 0.06$ & $2.37 \pm 0.11$ \\
\hline 4 & Riverside soil & $2.31 \pm 0.05$ & $0.83 \pm 0.05$ & $1.47 \pm 0.11$ & $1.35 \pm 0.02$ & $0.45 \pm 0.02$ & $0.89 \pm 0.04$ & $3.52 \pm 0.02$ & $0.87 \pm 0.07$ & $2.65 \pm 0.09$ \\
\hline 6 & & $3.11 \pm 0.11$ & $1.11 \pm 0.06$ & $2.00 \pm 0.17$ & $1.21 \pm 0.04$ & $0.41 \pm 0.04$ & $0.81 \pm 0.08$ & $4.41 \pm 0.03$ & $0.94 \pm 0.09$ & $3.46 \pm 0.12$ \\
\hline 2 & & $2.05 \pm 0.07$ & $0.71 \pm 0.03$ & $1.35 \pm 0.11$ & $2.06 \pm 0.02$ & $0.65 \pm 0.06$ & $1.41 \pm 0.08$ & $4.11 \pm 0.03$ & $0.67 \pm 0.06$ & $3.44 \pm 0.09$ \\
\hline 4 & Pond water & $2.71 \pm 0.05$ & $0.93 \pm 0.04$ & $1.77 \pm 0.09$ & $1.81 \pm 0.03$ & $0.52 \pm 0.04$ & $1.28 \pm 0.07$ & $4.46 \pm 0.04$ & $0.76 \pm 0.08$ & $3.71 \pm 0.12$ \\
\hline 6 & & $3.65 \pm 0.07$ & $1.14 \pm 0.09$ & $2.51 \pm 0.16$ & $1.55 \pm 0.03$ & $0.48 \pm 0.06$ & $1.07 \pm 0.08$ & $5.85 \pm 0.04$ & $0.81 \pm 0.04$ & $5.06 \pm 0.08$ \\
\hline 2 & & $1.11 \pm 0.03$ & $0.53 \pm 0.03$ & $0.57 \pm 0.06$ & $2.03 \pm 0.01$ & $0.63 \pm 0.04$ & $1.29 \pm 0.06$ & $1.66 \pm 0.02$ & $0.54 \pm 0.06$ & $1.12 \pm 0.08$ \\
\hline 4 & Sea water & $1.31 \pm 0.03$ & $0.62 \pm 0.04$ & $0.68 \pm 0.07$ & $1.52 \pm 0.02$ & $0.47 \pm 0.03$ & $1.04 \pm 0.05$ & $1.95 \pm 0.04$ & $0.61 \pm 0.05$ & $1.35 \pm 0.09$ \\
\hline 6 & & $2.21 \pm 0.07$ & $1.00 \pm 0.02$ & $1.21 \pm 0.09$ & $1.31 \pm 0.03$ & $0.43 \pm 0.04$ & $0.86 \pm 0.07$ & $2.28 \pm 0.03$ & $0.68 \pm 0.04$ & $1.61 \pm 0.07$ \\
\hline
\end{tabular}


Table 3

Roughness and section analyses of the environmental stressed textile fibres. The values for the surface roughnesses and heights of the fibres, calculated over 50 and 40 different areas, respectively, are given as the mean \pm standard deviation.

\begin{tabular}{|c|c|c|c|c|c|c|c|}
\hline \multirow{2}{*}{ Time (weeks) } & \multirow{2}{*}{ Environment } & \multicolumn{2}{|c|}{ Cotton } & \multicolumn{2}{|c|}{ Wool } & \multicolumn{2}{|c|}{ Viscose } \\
\hline & & $R_{r m s}(\mathrm{~nm})$ & $\mathrm{R}_{\mathrm{z}}(\mathrm{nm})$ & $R_{r m s}(\mathrm{~nm})$ & $\mathrm{R}_{\mathrm{z}}(\mathrm{nm})$ & $R_{r m s}(\mathrm{~nm})$ & $\mathrm{R}_{\mathrm{z}}(\mathrm{nm})$ \\
\hline 0 & - & $76.6 \pm 4.5$ & $156.1 \pm 11.3$ & $311.6 \pm 49.6$ & $848.2 \pm 147.1$ & $139.4 \pm 8.2$ & $645.5 \pm 30.1$ \\
\hline 2 & & $113.0 \pm 8.0$ & $413.2 \pm 50.4$ & $360.6 \pm 10.8$ & $1008.8 \pm 36.4$ & $291.4 \pm 22.1$ & $1055.4 \pm 41.4$ \\
\hline 4 & Loam soil & $225.5 \pm 6.4$ & $606.1 \pm 87.2$ & $405.2 \pm 13.2$ & $1119.3 \pm 39.7$ & $422.9 \pm 34.2$ & $1290.4 \pm 38.1$ \\
\hline 6 & & $334.3 \pm 15.5$ & $1062.9 \pm 99.8$ & $430.6 \pm 10.1$ & $1190.6 \pm 16.1$ & $530.6 \pm 27.1$ & $1480.8 \pm 49.9$ \\
\hline 2 & & $160.7 \pm 6.7$ & $597.6 \pm 21.5$ & $337.8 \pm 20.4$ & $953.9 \pm 59.6$ & $237.0 \pm 25.9$ & $867.8 \pm 77.4$ \\
\hline 4 & Riverside soil & $384.0 \pm 27.0$ & $1116.5 \pm 26.9$ & $369.2 \pm 22.2$ & $1004.1 \pm 40.6$ & $351.3 \pm 32.9$ & $1128.2 \pm 86.8$ \\
\hline 6 & & $501.3 \pm 47.1$ & $1638.7 \pm 88.9$ & $391.1 \pm 24.7$ & $1100.7 \pm 28.7$ & $442.8 \pm 21.6$ & $1320.9 \pm 52.4$ \\
\hline 2 & & $122.3 \pm 7.9$ & $470.3 \pm 28.5$ & $312.1 \pm 26.6$ & $861.3 \pm 80.1$ & $207.4 \pm 17.2$ & $716.9 \pm 102.05$ \\
\hline 4 & Pond water & $307.7 \pm 16.5$ & $810.7 \pm 44.6$ & $322.6 \pm 26.2$ & $890.3 \pm 44.4$ & $272.8 \pm 18.9$ & $956.6 \pm 50.8$ \\
\hline 6 & & $420.1 \pm 40.1$ & $1364.1 \pm 140.1$ & $331.8 \pm 7.1$ & $932.5 \pm 33.2$ & $340.9 \pm 24.3$ & $1097.4 \pm 38.2$ \\
\hline 2 & & $213.5 \pm 17.4$ & $867.3 \pm 19.5$ & $323.7 \pm 19.5$ & $889.6 \pm 39.1$ & $363.0 \pm 28.1$ & $1249.3 \pm 39.6$ \\
\hline 4 & Sea water & $487.3 \pm 41.4$ & $1433.6 \pm 34.1$ & $333.4 \pm 26.7$ & $935.8 \pm 40.3$ & $499.3 \pm 20.4$ & $1431.9 \pm 41.1$ \\
\hline 6 & & $716.5 \pm 74.9$ & $1938.5 \pm 120.4$ & $356.3 \pm 17.4$ & $1013.2 \pm 30.8$ & $637.6 \pm 35.7$ & $1597.8 \pm 42.2$ \\
\hline
\end{tabular}




\section{Figure captions}

Fig. 1. (a) Section analysis on the AFM height image (top) and corresponding profile (bottom) for the wool fibre stressed with loam soil for 2 weeks. The two dashed lines on the AFM height image correspond to the dashed lines shown on the profile. (b) 3D AFM image corresponding to the AFM height image shown in (a).

Fig. 2. AFM height images of cotton fibre exposed to loam and riverside soils, and pond and sea waters for 2 and 6 weeks. The fibre not exposed to any environmental stress ( 0 weeks) was used as a control. The scan size of each image is $10 \mu \mathrm{m} \times 10$ $\mu \mathrm{m}$. The bar on the right hand side of each AFM image shows the maximum height of the image.

Fig. 3. AFM height images of viscose fibre exposed to loam and riverside soils, and pond and sea waters for 2 and 6 weeks. The fibre not exposed to any environmental stress ( 0 weeks $)$ was used as a control. The scan size of each image is $10 \mu \mathrm{m} \times 10$ $\mu \mathrm{m}$. The bar on the right hand side of each AFM image shows the maximum height of the image.

Fig. 4. AFM height images of wool fibre exposed to loam and riverside soils, and pond and sea waters for 2 and 6 weeks. The fibre not exposed to any environmental stress $(0$ weeks $)$ was used as a control. The scan size of each image is $10 \mu \mathrm{m} \times 10$ $\mu \mathrm{m}$. The bar on the right hand side of each AFM image shows the maximum height of the image. 


\section{Figures}
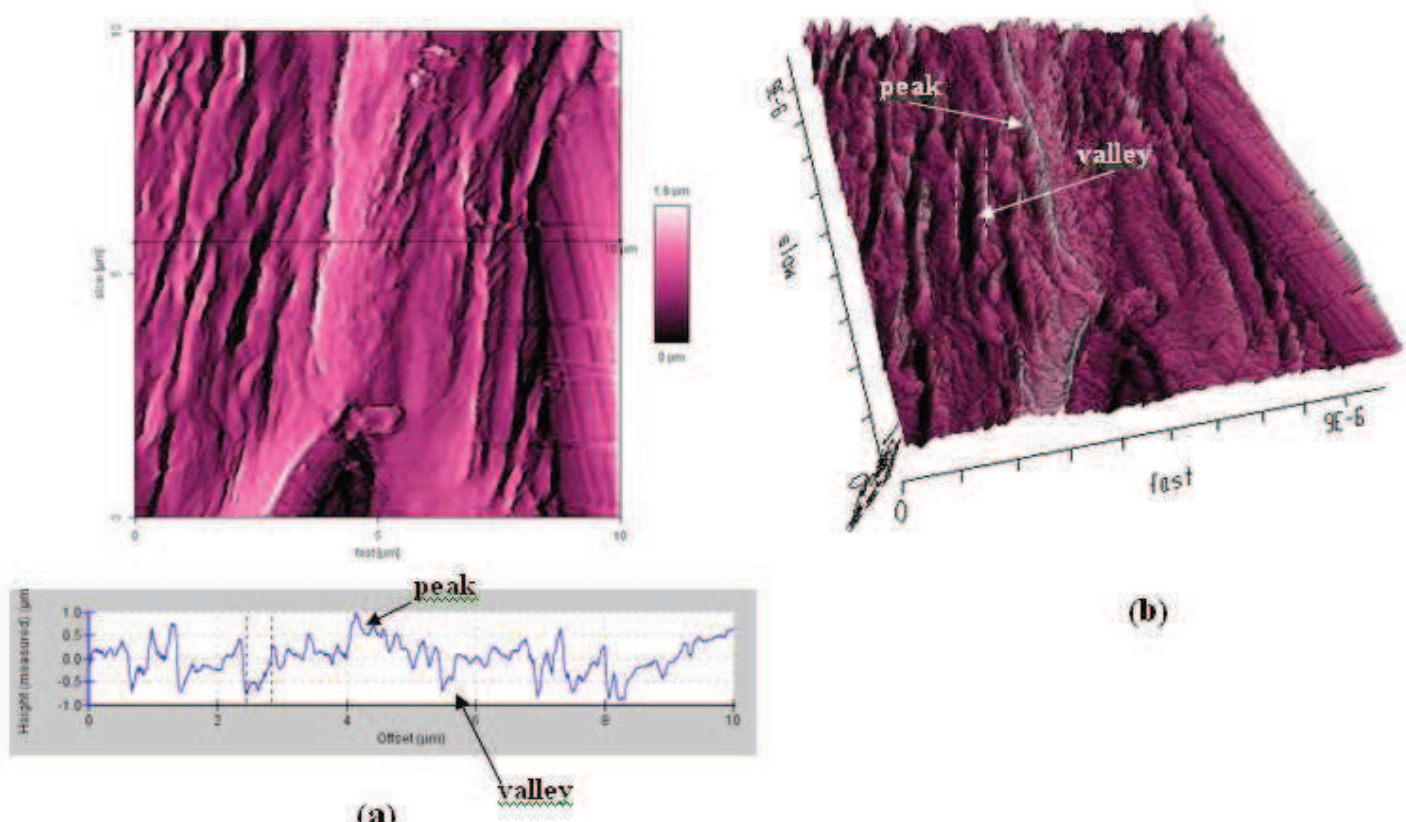

(b)

Fig. 1. 


\section{Control}

( 0 w eeks)
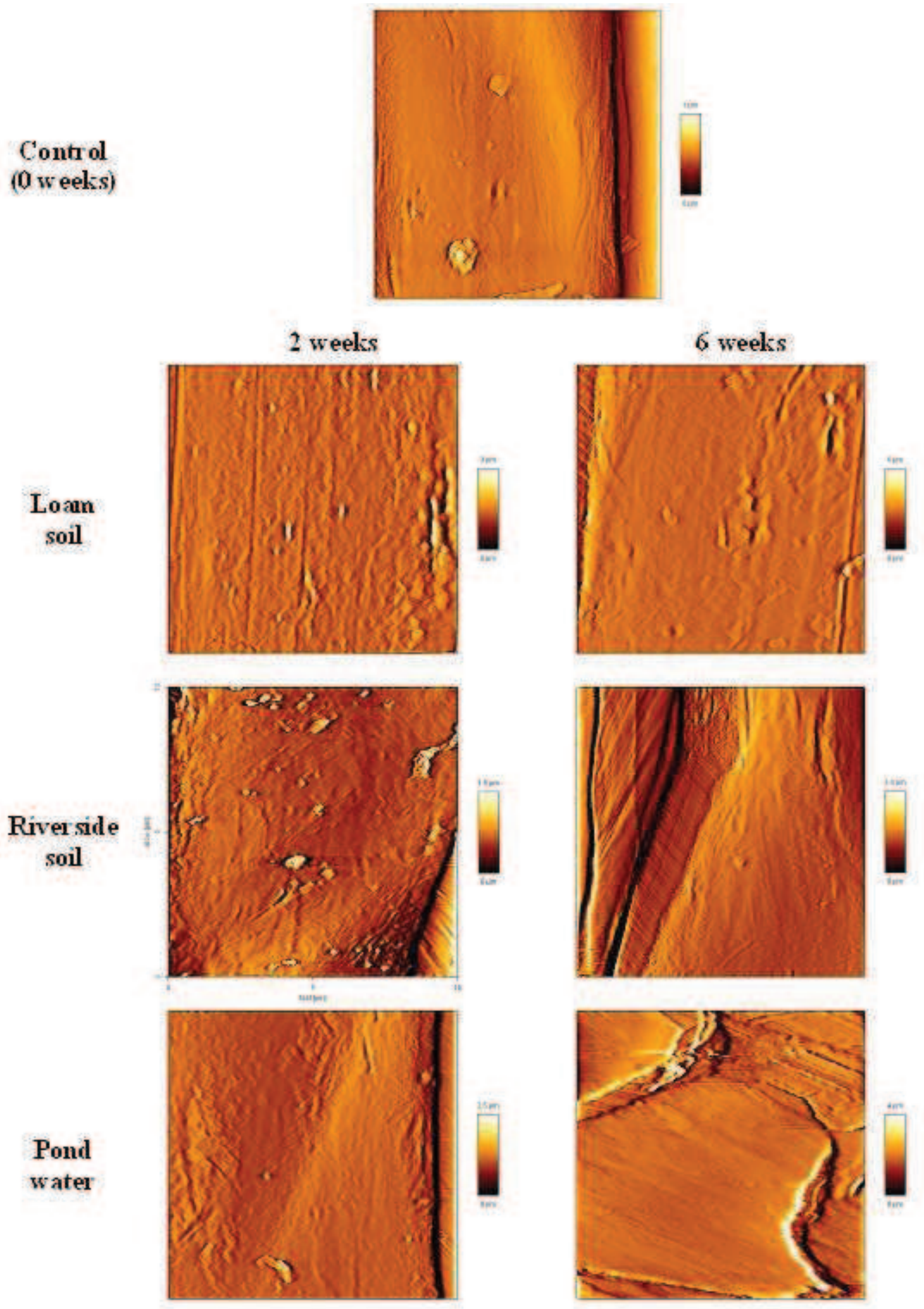

Fig. 2. 
Control

(0w eeks)
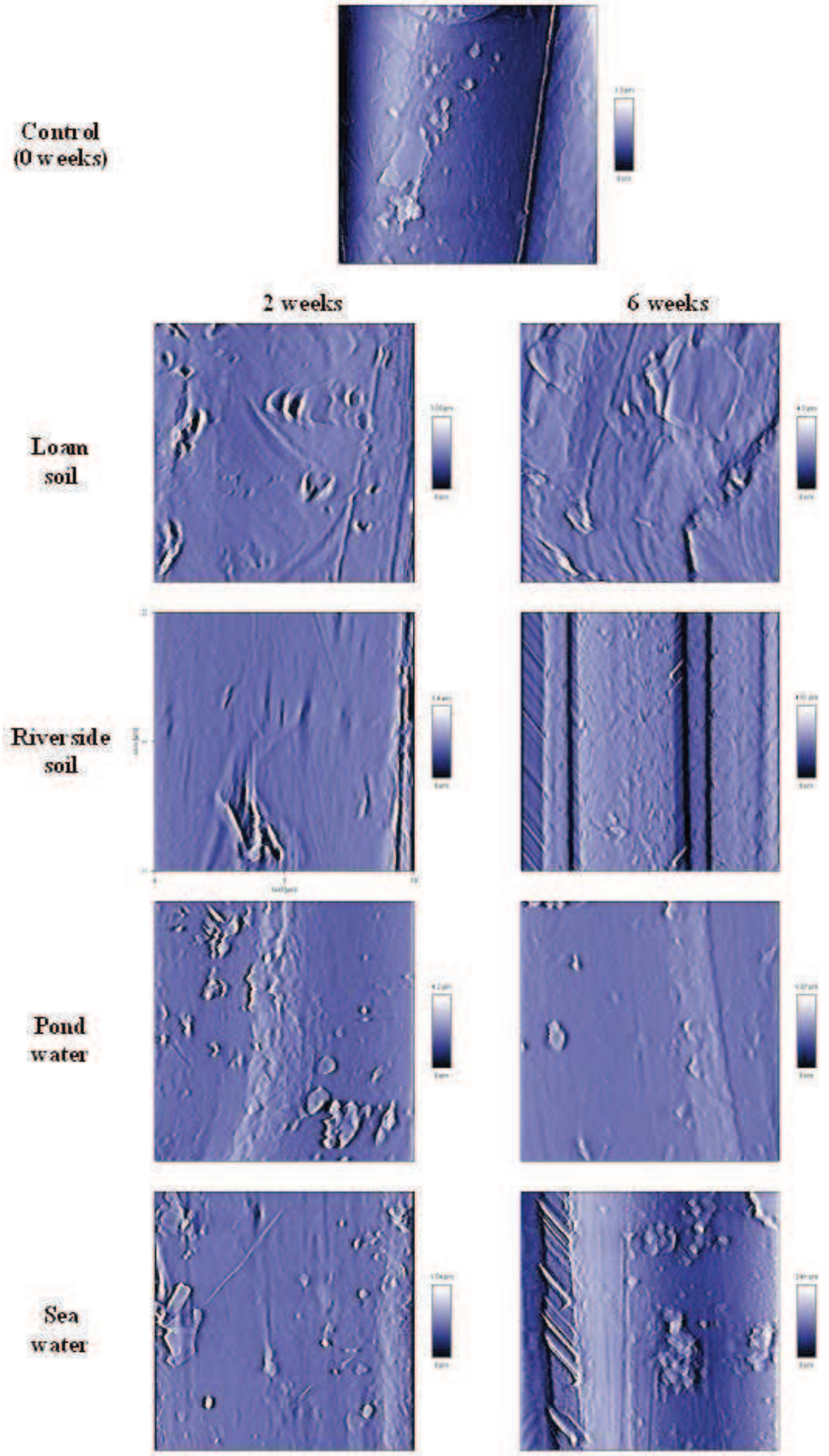

Fig. 3. 
Control

(0 w eeks)
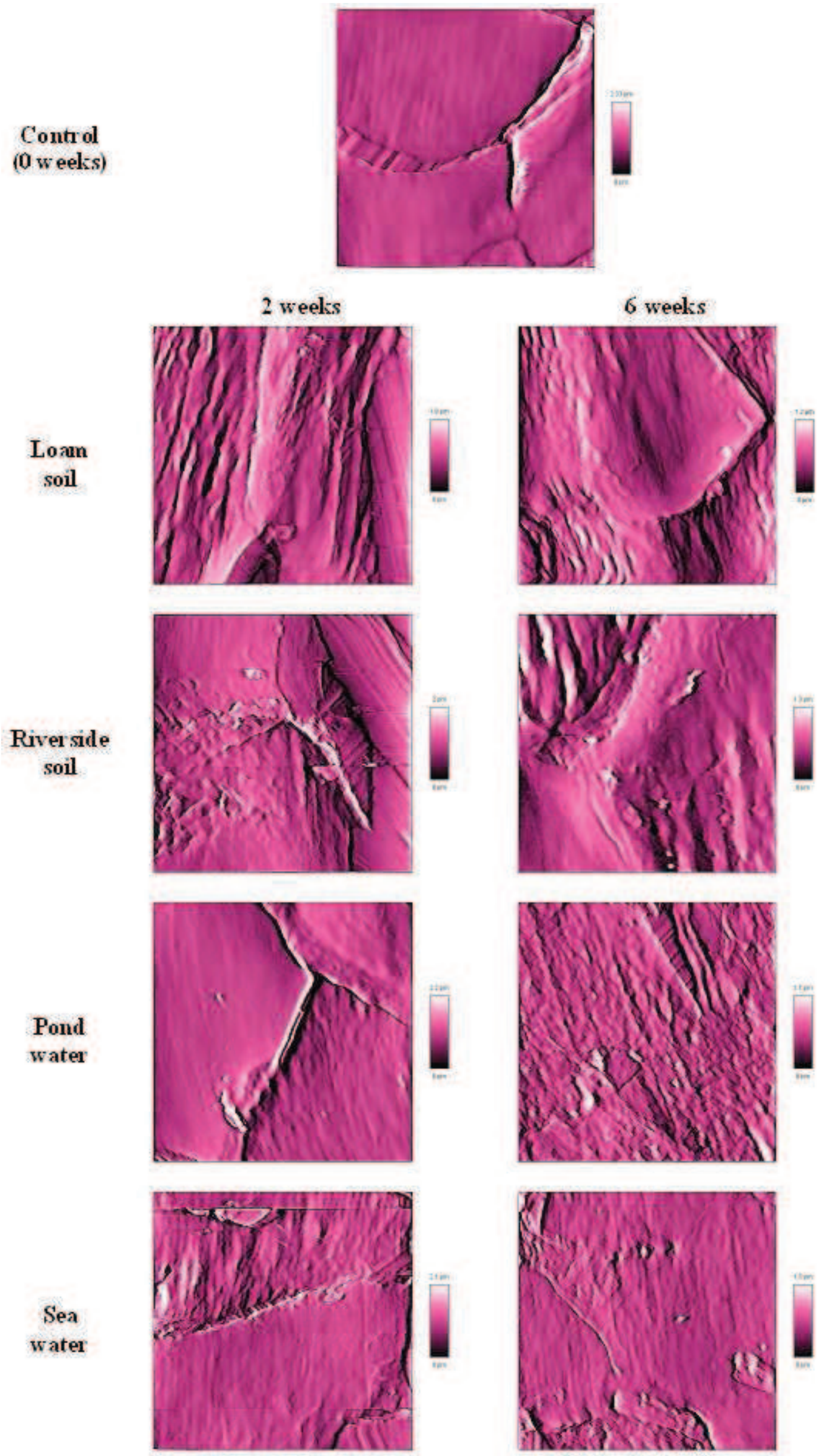

Fig. 4. 


\section{References}

[1] J. Robertson and M. Grieve, Forensic examination of fibres, $2^{\text {nd }}$ ed., Taylor \& Francis Ltd., London, 1999.

[2] M.C. Grieve and T. Biermann, The population of coloured textile fibres on outdoor surfaces, Science \& Justice 37 (1997) 231-239.

[3] E.G. Bartick, J.V. Miller, Forensic fiber analysis by microscopical Raman spectroscopy, in: Proceedings of the $15^{\text {th }}$ International Association of Forensic Science Meeting, Los Angeles, 1999, pp. 149-150.

[4] G. Binning, C.F. Quate, C. Gerber, Atomic force microscope, Phys. Rev. Lett. 56 (1986) 930-933.

[5] J.R. Smith, A quantitative method for analysing AFM images of the outer surfaces of human hair, J. Microscop. 191 (1998) 223-228.

[6] S.P. Gurden, V.F. Monteiro, E. Longo, M.M. Ferreira, Quantitative analysis and classification of AFM images of human hair, J. Microscop. 215 (2004) 13-23.

[7] S. Kasas, A. Khanmy-Vital, G. Dietler, Examination of line crossings by atomic force microscopy, Forensic Sci. Int. 119 (2001) 290-298.

[8] C. Bersellini, L. Garofano, M. Giannetto, F. Lusardi, G. Mori, Development of latent fingerprints on metallic surfaces using electropolymerization processes, J. Forensic Sci. 46 (2001) 871-877.

[9] Y. Chen, J. Cai, Membrane deformation of unfixed erythrocytes in air with time lapse investigated by tapping mode atomic force microscopy, Micron 37 (2006) 339-346.

[10] G. Poletti, F. Orsini, A. Raffaele-Addamo, C. Riccardi, E. Selli, Cold plasma treatment of PET fabrics: AFM surface morphology characterisation, Appl. Surf. Science 219 (2003) 311-316.

[11] Q. Wang, X. Fan, W. Gao, J. Chen, Characterization of bioscoured cotton fabrics using FT-IR ATR spectroscopy and microscopy techniques, Carbohyd. Res. 341 (2006) 2170-2175. 